ANNALES

POLONICI MATHEMATICI

$95.3(2009)$

\title{
On the real analyticity of the scattering operator for the Hartree equation
}

\author{
by Changxing Miao (Beijing), Haigen Wu (Beijing and Jiaozuo) and \\ JUNYONG ZHANG (Beijing)
}

\begin{abstract}
We study the real analyticity of the scattering operator for the Hartree equation $i \partial_{t} u=-\Delta u+u\left(V *|u|^{2}\right)$. To this end, we exploit interior and exterior cut-off in time and space, together with a compactness argument to overcome difficulties which arise from absence of good properties as for the Klein-Gordon equation, such as the finite speed of propagation and ideal time decay estimate. Additionally, the method in this paper allows us to simplify the proof of analyticity of the scattering operator for the nonlinear Klein-Gordon equation with cubic nonlinearity.
\end{abstract}

1. Introduction. This paper is devoted to the proof of the real analyticity of the scattering operator for the Hartree equation

$$
\left\{\begin{array}{l}
i \partial_{t} u=-\Delta u+u\left(V *|u|^{2}\right), \quad(t, x) \in \mathbb{R} \times \mathbb{R}^{3}, \\
u(0)=u_{0} \in H^{1}\left(\mathbb{R}^{3}\right) .
\end{array}\right.
$$

Here $u(t, x)$ is a complex-valued function defined in $\mathbb{R}^{1+3}, V(x)$, called a potential, is a real-valued radial function defined in $\mathbb{R}^{3}$, and $*$ denotes convolution in $\mathbb{R}^{3}$. Under suitable assumptions on $V$, Ginibre-Velo [7] developed the scattering theory of the equation (1.1) in the energy space $H^{1}$. Attempting to study the (complex) analyticity of the scattering operator is in vain because $\bar{u}$ is not analytic even if $u$ is. However, following W. Strauss's suggestion (private communication), we can study the real analyticity which is still an interesting issue.

Let $u=\varphi(t, x)+i \psi(t, x), u_{0}=\varphi_{0}(x)+i \psi_{0}(x)$, where $\varphi(t, x), \psi(t, x)$, $\varphi_{0}(x), \psi_{0}(x)$ are real-valued functions defined in $\mathbb{R} \times \mathbb{R}^{3}$ or $\mathbb{R}^{3}$. Then the integral form of equation (1.1)

$$
u(t)=e^{i t \Delta} u_{0}-i \int_{0}^{t} e^{i(t-s) \Delta}\left(\left(V *|u|^{2}\right) u\right)(s) d s
$$

2000 Mathematics Subject Classification: 35P25, 35Q55.

Key words and phrases: Hartree equation, real analyticity, scattering operator, compactness. 
can be rewritten as

$$
\begin{aligned}
\left(\begin{array}{l}
\varphi \\
\psi
\end{array}\right) & =\left(\begin{array}{cc}
\cos t \Delta & -\sin t \Delta \\
\sin t \Delta & \cos t \Delta
\end{array}\right)\left(\begin{array}{l}
\varphi_{0} \\
\psi_{0}
\end{array}\right) \\
& +\int_{0}^{t}\left(\begin{array}{cc}
\sin (t-s) \Delta & \cos (t-s) \Delta \\
-\cos (t-s) \Delta & \sin (t-s) \Delta
\end{array}\right)\left(\begin{array}{l}
\varphi \\
\psi
\end{array}\right)\left(V *\left(\varphi^{2}+\psi^{2}\right)\right)(s) d s
\end{aligned}
$$

Setting

$$
U(t)=\left(\begin{array}{l}
\varphi(t) \\
\psi(t)
\end{array}\right) \quad \text { and } \quad U_{0}=\left(\begin{array}{l}
\varphi_{0} \\
\psi_{0}
\end{array}\right)
$$

we can transform (1.3) into

$$
\mathcal{N}(t) U_{0}:=U(t)=G(t) U_{0}-\int_{0}^{t} \Delta^{-1} G^{\prime}(t-s)\left(V * \mid U^{2}\right) U(s) d s
$$

where

$$
G(t)=\left(\begin{array}{cc}
\cos t \Delta & -\sin t \Delta \\
\sin t \Delta & \cos t \Delta
\end{array}\right)
$$

is the unitary group associated with the equation (1.1).

First, we recall the decay estimate and Strichartz estimates in the context of Schrödinger equation (see [6], [9], [11]).

Definition 1.1. A pair $(q, r)$ is admissible, denoted by $(q, r) \in \Lambda$, if $r \in[2,6]$ and $q$ satisfies

$$
\frac{2}{q}=\delta(r):=3\left(\frac{1}{2}-\frac{1}{r}\right)
$$

LEMMA 1.1. Let $S(t)=e^{i t \Delta}$. Then:

(1) the $L^{r^{\prime}}-L^{r}$ decay estimate

$$
\|S(t) \varphi\|_{r} \leq C|t|^{-\delta(r)}\|\varphi(x)\|_{r^{\prime}}
$$

holds for $2 \leq r \leq \infty$;

(2) the Strichartz estimates

$$
\begin{aligned}
& \|S(t) u\|_{L^{q}\left(\mathbb{R}, L^{r}\left(\mathbb{R}^{3}\right)\right)} \leq C\|u\|_{2},
\end{aligned}
$$

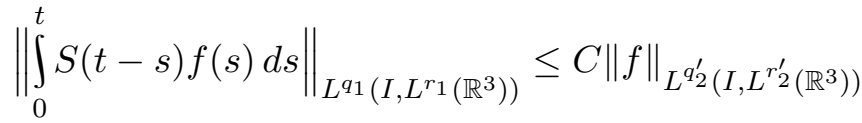

hold for any interval $I \subset \mathbb{R}$, and for any admissible pairs $(q, r),\left(q_{j}, r_{j}\right)$ $\in \Lambda, j=1,2$. 
REMARK 1.1. Lemma 1.1 still holds for the propagators $G(t)$ and $\Delta^{-1} G^{\prime}(t)$ by the Euler formulae

$$
\cos t \Delta=\frac{e^{i t \Delta}+e^{-i t \Delta}}{2}, \quad \sin t \Delta=\frac{e^{i t \Delta}-e^{-i t \Delta}}{2} .
$$

Let $B$ be a Banach space, and write

$$
\left(\begin{array}{l}
u \\
v
\end{array}\right) \in B \Leftrightarrow\left\|\left(\begin{array}{l}
u \\
v
\end{array}\right)\right\|_{B}=\|u\|_{B}+\|v\|_{B}<\infty .
$$

Throughout this paper, the symbol $C$ denotes a constant which may be different from line to line, and $C(*)$ denotes a constant which only depends on the parameter $*$.

Define the wave operator $W_{ \pm}: U_{ \pm} \mapsto U_{0}$ as follows: for any $U_{ \pm} \in H^{1}$, there exists $U_{0} \in H^{1}$ such that

$$
\left\|G(t) U_{ \pm}-\mathcal{N}(t) U_{0}\right\|_{H^{1}} \rightarrow 0 \quad \text { as } t \rightarrow \pm \infty .
$$

When the wave operators $W_{ \pm}$are invertible, we can define the scattering operator as $S=W_{+}^{-1} \circ W_{-}: U_{-} \mapsto U_{+}$.

Set

$$
X=C\left(\mathbb{R}, H^{1}\left(\mathbb{R}^{3}\right)\right) \cap \bigcap_{(q, r) \in \Lambda} L^{q}\left(\mathbb{R}, H_{r}^{1}\left(\mathbb{R}^{3}\right)\right) .
$$

Ginibre-Velo established a complete scattering theory in energy space provided that the potential $V$ satisfies the following assumption:

(H1) $V$ is a real function and $V \in L^{p_{1}}+L^{p_{2}}$ for some $p_{1}, p_{2}$ satisfying

$$
1<p_{2} \leq p_{1}<3 / 2 \text {. }
$$

(H2) $V$ is radial and nonincreasing, i.e., $V(x)=v(r)$ where $v$ is nonincreasing in $\mathbb{R}^{+}$. Furthermore, for some $\alpha \geq 2, v$ satisfies the following condition:

$\left(A_{\alpha}\right)$ There exist $a>0$ and $A_{\alpha}>0$ such that

$$
v\left(r_{1}\right)-v\left(r_{2}\right) \geq \frac{A_{\alpha}}{\alpha}\left(r_{2}^{\alpha}-r_{1}^{\alpha}\right) \quad \text { for } 0<r_{1}<r_{2} \leq a .
$$

In particular, the wave operator $W_{ \pm}$and the scattering operator $S$ are bounded and continuous from $H^{1}$ to $H^{1}$.

Our main result is

THEOREM 1.1. Let $V$ satisfy the assumptions (H1) and (H2). Then the operators $W_{ \pm}$and $S$ are analytic from $H^{1}$ to $H^{1}$.

The proof of Theorem 1.1 depends on the following theorem:

THEOREM 1.2. Let $U_{0} \in H^{1}$ and $U(t)$ be the unique solution of (1.4) in $X$. Then the map $U: U_{0} \mapsto U\left(U_{0}\right)$ is analytic from $H^{1}$ to $X$. 
For the nonlinear Klein-Gordon equation with cubic nonlinearity, using the contraction mapping principle, Baez-Zhou [2] proved the analyticity of the scattering operator on a neighborhood of zero in the space of finiteenergy Cauchy data, $H^{1} \oplus L^{2}\left(\mathbb{R}^{3}\right)$. Kumlin [10] generalized that result to the entire energy space by means of the Fredholm alternative theorem. The proof in [10] depends on the following two nice properties of the linear KleinGordon equation:

(1) $L^{p}-L^{p^{\prime}}$ estimates stated in the following proposition.

Proposition 1.1 ([4], [10]). Let

$$
\begin{aligned}
& K(t)=\frac{\sin t\left(-\Delta+m^{2}\right)^{1 / 2}}{\left(-\Delta+m^{2}\right)^{1 / 2}} \\
& 1<p \leq 2 \leq p^{\prime}, 1 / p+1 / p^{\prime}=1, \sigma:=1 / 2-1 / p^{\prime} \text { and } 0 \leq \theta \leq 1 \text {. If } \\
& (n+1+\theta) \sigma \leq 1+s-s^{\prime}, \text { then } \\
& \|K(t) g\|_{W^{s^{\prime}, p^{\prime}}} \leq k(t)\|g\|_{W^{s, p}}, \quad t \geq 0,
\end{aligned}
$$

where

$$
k(t)= \begin{cases}t^{-(n-1-\theta) \sigma}, & 0<t<1 \\ t^{-(n-1+\theta) \sigma}, & 1 \leq t\end{cases}
$$

For suitable $p$ and $\theta$, we have $k \in L^{1}(\mathbb{R})$. In particular,

$$
\|K(t) g\|_{H^{1}} \leq C\|g\|_{L^{2}} .
$$

These estimates are crucial in the proofs of Steps 1 and 3 in [10].

(2) The finite speed of propagation.

The finite speed of propagation of the solution of a linear wave equation means that for $t \in[-T, T], T<\infty$,

$$
\left\|\int_{0}^{t} K(t-s) u(s) d s\right\|_{L^{6}(|x|>R)} \sim\left\|\int_{0}^{t} K(t-s) \eta_{R} u(s) d s\right\|_{L^{6}\left(\mathbb{R}^{3}\right)} .
$$

Here the cut-off function $\eta_{R}(x)$ defined in (1.15) below commutes with the group $K(t)$ in some sense, which plays an important role in the proof of analyticity (see Step 2 in [10]).

The arguments in this paper still apply the Fredholm alternative theorem together with the analytic version of the implicit function theorem (cf. [2, $3,10])$. However, we have to overcome some difficulties arising from loss of the good properties (1) and (2) for the Schrödinger equation. Our major innovations are as follows: Compared with $k(t)$ in (1.10), the kernel $|t|^{-\delta(r)}$ in (1.6) is not in any $L^{p}(\mathbb{R}), 1 \leq p \leq \infty$, and the Hardy-Littlewood-Sobolev inequality cannot supply any decay. A new approach to the singular kernel 
is the double localization in time

$$
|t-s|^{-\delta(r)} \chi_{\{|s| \leq T / 2\}} \chi_{\{|t|>T\}}, \quad|t-s|^{-\delta(r)} \chi_{\{|s|>T / 2\}} \chi_{\{|t|>T\}},
$$

which together with other techniques helps us to get time decay; here $\chi_{A}$ denotes the characteristic function of the interval $A$. On the other hand, modifying (1.11) by introducing double (interior and exterior) cut-off in space

$$
\begin{aligned}
& \left\|\int_{0}^{t} \Delta^{-1} G^{\prime}(t-s)\left(\left(V *|U|^{2}\right) \xi_{R} \Psi_{j}\right)(s) d s\right\|_{L^{4}\left(|t| \leq T, H_{3}^{1}(|x|>M)\right)}, \\
& \left\|\int_{0}^{t} \Delta^{-1} G^{\prime}(t-s)\left(\left(V *|U|^{2}\right) \eta_{R} \Psi_{j}\right)(s) d s\right\|_{L^{4}\left(|t| \leq T, H_{3}^{1}\left(\mathbb{R}^{3}\right)\right)},
\end{aligned}
$$

we obtain decay estimates and overcome the above difficulties by means of a compactness principle; here

$$
\xi_{R} \in C_{0}^{\infty}\left(\mathbb{R}^{3}\right), \quad \xi_{R}(x)=\left\{\begin{array}{ll}
1 & \text { if }|x| \leq R, \\
0 & \text { if }|x|>2 R,
\end{array} \quad \eta_{R}(x)=1-\xi_{R}(x) .\right.
$$

Based on these decay estimates and the arguments in Kumlin [10], we can prove Theorem 1.1 by applying the approximation theorem for analytic operator sequences (cf. [8]). However, it is worth mentioning that we make use of compactness arguments and the definition of Fréchet derivative to avoid repeating the argument of global time space integrability,which makes the proof of Theorem 1.1 more concise.

The paper is organized as follows: In Section 2 we give the proof of Theorem 1.2; Section 3 is devoted to the proof of a key lemma, which constitutes the main part of this paper; finally, we supply a brief derivation of Theorem 1.1 in Section 4.

2. Proof of Theorem 1.2. We need the following analytic version of the implicit function theorem.

Lemma 2.1 ([3]). Suppose that $X, Y, Z$ are Banach spaces and $Q$ is an open neighborhood of the point $(x, y) \in X \times Y$. Suppose that $f: Q \rightarrow Z$ is analytic, $f(x, y)=0$ and $D_{2} f(x, y): X \rightarrow Z$ has a left inverse, where $D_{2}$ indicates the Fréchet derivative with respect to the second variable. Then for some open set $P$ containing $x$, there exists a unique analytic function $g: P \rightarrow Y$ such that $g(x)=y$ and $f\left(x^{\prime}, g\left(x^{\prime}\right)\right)=0$ for all $x^{\prime} \in P$.

For $U_{0} \in H^{1}$ and $\Psi \in X$, we set

$$
R\left(U_{0}, \Psi\right)=G(t) U_{0}-\int_{0}^{t} \Delta^{-1} G^{\prime}(t-s)\left(\left(V *|\Psi|^{2}\right) \Psi\right)(s) d s-\Psi(t) .
$$


Since $R$ is linear in $U_{0}$ and multilinear in $\Psi$, we know that $R: H^{1} \times X \rightarrow X$ is analytic by the nonlinear estimate in [7]

$$
\left\|\int_{0}^{t} \Delta^{-1} G^{\prime}(t-s)\left(\left(V *|\Psi|^{2}\right) \Psi\right)(s) d s\right\|_{X}<\infty, \quad \forall \Psi \in X .
$$

On the other hand, $R\left(U_{0}, U\right)=0$ by (1.4). Hence it suffices to prove the invertibility of

$$
D_{2} R\left(U_{0}, U\right): X \rightarrow X
$$

for each $U_{0} \in H^{1}$. By the open mapping theorem, we only need to prove that $D_{2} R\left(U_{0}, U\right)$ is injective and surjective.

For $U_{0} \in H^{1}$ and $\Psi \in X$, one has

$$
\begin{aligned}
D_{2} R\left(U_{0}, U\right)(\Psi)(t)= & -2 \int_{0}^{t} \Delta^{-1} G^{\prime}(t-s)(V *(\Psi U)) \Psi(s) d s \\
& -\int_{0}^{t} \Delta^{-1} G^{\prime}(t-s)\left(V *|U|^{2}\right) \Psi(s) d s-\Psi(t) .
\end{aligned}
$$

(1) The injectivity of $D_{2} R\left(U_{0}, U\right)$. For simplicity, we always assume that $V \in L^{p}$. Let $D_{2} R\left(U_{0}, U\right) \Psi=0$. Then

$$
\begin{aligned}
\|\Psi\|_{r} & \leq C \int_{0}^{t}|t-s|^{-\delta(r)}\left(\|(V *(U \Psi)) U(s)\|_{r^{\prime}}+\left\|\left(V *|U|^{2}\right) \Psi(s)\right\|_{r^{\prime}}\right) d s \\
& \leq 2 C \int_{0}^{t}|t-s|^{-\delta(r)}\|V\|_{p}\|U\|_{l}^{2}\|\Psi\|_{r} d s
\end{aligned}
$$

where $2=1 / p+2 / l+2 / r$.

For every $p \in(1,3 / 2)$, we can take $l=r \in(3,4)$ such that

$$
\|\Psi\|_{r} \leq C\|V\|_{p}\|U\|_{L^{\infty} H^{1}}^{2} \int_{0}^{t}|t-s|^{-\delta(r)}\|\Psi\|_{r} d s .
$$

For each $t \in(0, T)$, one easily verifies that by (2.4),

$$
\|\Psi(t)\|_{r} \leq C t^{1-\delta(r)} \operatorname{ess~sup}_{s \in(0, t)}\|\Psi(s)\|_{r} .
$$

We choose $T$ small enough such that

$$
\underset{t \in(0, T)}{\operatorname{ess} \sup }\|\Psi(t)\|_{r} \leq \frac{1}{2} \operatorname{ess~sup}_{s \in(0, T)}\|\Psi(s)\|_{r} .
$$

This implies that $\Psi \equiv 0$ for a.e. $t \in[0, T]$, for some $T>0$. Repeating this process on $(n T, n T+T), n \in \mathbb{Z}$, we have $\Psi \equiv 0$ for a.e. $(x, t) \in \mathbb{R}^{3+1}$. 
(2) The surjectivity of $D_{2} R\left(U_{0}, U\right)$. Setting

$$
\begin{aligned}
\mathcal{T}_{U_{0}} \Psi(t)= & -\int_{0}^{t} \Delta^{-1} G^{\prime}(t-s)\left(\left(V *|U|^{2}\right) \Psi\right)(s) d s \\
& -2 \int_{0}^{t} \Delta^{-1} G^{\prime}(t-s)((V *(U \Psi)) U)(s) d s,
\end{aligned}
$$

we have $D_{2} R\left(U_{0}, U\right)=\mathcal{T}_{U_{0}}-I$. By the Fredholm alternative, one could attempt to show that $\mathcal{T}_{U_{0}}$ is a compact operator from $X$ to $X$ since $\mathcal{T}_{U_{0}}-I$ is injective. However, $\mathcal{T}_{U_{0}}$ may not be compact. But for our goal, it suffices to show that $\mathcal{T}_{U_{0}}^{2}$ is compact. In fact, since

$$
\mathcal{T}_{U_{0}}^{2}-I=\left(\mathcal{T}_{U_{0}}-I\right)\left(\mathcal{T}_{U_{0}}+I\right)
$$

and $\mathcal{T}_{U_{0}}+I$ is also injective, the Fredholm theorem still works. Therefore (2.6) implies the surjectivity of $\mathcal{T}_{U_{0}}-I$.

Concerning the trilinear form

$$
\mathcal{B}\left(\Psi_{1}, \Psi_{2}, \Psi_{3}\right):=-\int_{0}^{t} \Delta^{-1} G^{\prime}(t-s)\left(V *\left(\Psi_{1} \Psi_{2}\right)\right) \Psi_{3}(s) d s,
$$

we have the following nonlinear estimate.

Lemma 2.2. For $\Psi_{j} \in X, j=1,2,3$, one has

$$
\left\|\mathcal{B}\left(\Psi_{1}, \Psi_{2}, \Psi_{3}\right)\right\|_{X} \lesssim \prod_{j=1,2,3}\left\|\Psi_{j}\right\|_{L^{4} H_{3}^{1}}
$$

Proof. Using the Strichartz estimates together with the Hölder inequality, we obtain

$$
\begin{aligned}
\| \int_{0}^{t} \Delta^{-1} G^{\prime}(t-s) & \left(V *\left(\Psi_{1} \Psi_{2}\right)\right) \Psi_{3}(s) d s \|_{X} \\
& \lesssim\left\|\left(V *\left(\Psi_{1} \Psi_{2}\right)\right) \Psi_{3}\right\|_{L^{4 / 3} H_{3 / 2}^{1}} \\
& \leq \sum_{\{i, j, k\}=\{1,2,3\}}\|V\|_{p}\left\|\Psi_{i}\right\|_{L^{4} H_{3}^{1}}\left\|\Psi_{j}\right\|_{L^{4} L^{\tilde{p}}}\left\|\Psi_{k}\right\|_{L^{4} L^{\widetilde{p}}} \\
& \lesssim \prod_{j=1,2,3}\left\|\Psi_{j}\right\|_{L^{4} H_{3}^{1}}
\end{aligned}
$$

where $\widetilde{p}=6 p /(4 p-3)$, and we have used the embedding $H_{3}^{1} \hookrightarrow L^{\widetilde{p}}$.

As a direct consequence of Lemma 2.2, we have

$$
\left\|\mathcal{T}_{U_{0}} \Psi(t)\right\|_{X} \lesssim\|\Psi\|_{L^{4} H_{3}^{1}}
$$


This implies that $\mathcal{T}_{U_{0}}: L^{4} H_{3}^{1} \rightarrow X$ is bounded. Since the composition of a compact operator and a bounded operator is still compact, it is enough to verify the following key lemma.

Lemma 2.3. Let $U_{0} \in H^{1}$. Then $\mathcal{T}_{U_{0}}: X \rightarrow L^{4} H_{3}^{1}$ is compact.

3. Proof of Lemma 2.3. Let $\left\{\Psi_{j}\right\}_{j=0}^{\infty}$ be uniformly bounded in $X$, i.e. $\left\|\Psi_{j}\right\|_{X} \leq C$ for constant $C>0$. We shall show that $\left\{\mathcal{T}_{U_{0}} \Psi_{j}\right\}_{j=0}^{\infty}$ has a Cauchy subsequence in $L^{4} H_{3}^{1}$. Our main tool is the Arzelà-Ascoli theorem, so it is necessary to localize both the time and space. Let $\xi_{R}, \eta_{R}$ be as in (1.15). The proof can be divided into five steps:

STEP 1. $\lim _{T \rightarrow \infty} \sup _{j \in \mathbb{N}}\left\|\mathcal{T}_{U_{0}} \Psi_{j}\right\|_{L^{4}\left(|t|>T, H_{3}^{1}\left(\mathbb{R}^{3}\right)\right)}=0 ;$

SteP 2. $\lim _{R \rightarrow \infty} \sup _{j \in \mathbb{N}}\left\|\mathcal{T}_{U_{0}}\left(\eta_{R} \Psi_{j}\right)\right\|_{L^{4}\left(|t| \leq T, H_{3}^{1}\left(\mathbb{R}^{3}\right)\right)}=0$ for all $T>0$;

STEP 3. $\lim _{M \rightarrow \infty} \sup _{j \in \mathbb{N}}\left\|\mathcal{T}_{U_{0}}\left(\xi_{R} \Psi_{j}\right)\right\|_{L^{4}\left(|t| \leq T, H_{3}^{1}(|x|>M)\right)}=0$ for all $T, R>0$;

STEP 4. $\left\{\mathcal{T}_{U_{0}}\left(\xi_{R} \Psi_{j}\right)\right\}_{j=0}^{\infty}$ has a Cauchy subsequence in $L^{4}\left(|t| \leq T, H_{3}^{1}(|x|\right.$ $\leq M)$ ) for all $T, R, M>0$;

STEP 5. A Cantor diagonal process.

For the sake of convenience, we first give some useful estimates.

Lemma 3.1. Let $\|U\|_{X} \leq C,\left\|\Psi_{j}\right\|_{X} \leq C$ and $V \in L^{p}\left(\mathbb{R}^{3}\right)$. Then for any $p \in(1,3 / 2)$ and sufficiently small $\delta>0$, we have

$$
\left\|\left(V *|U|^{2}\right) \Psi_{j}\right\|_{2} \leq C, \quad\left\|\left(V *|U|^{2}\right) \Psi_{j}\right\|_{\dot{H}^{\delta}} \leq C .
$$

Proof. For any $p \in(1,3 / 2)$, by the Sobolev embedding theorem,

$$
\left\|\left(V *|U|^{2}\right) \Psi_{j}\right\|_{2} \leq\|V\|_{p}\|U\|_{r}^{2}\left\|\Psi_{j}\right\|_{r} \leq\|V\|_{p}\|U\|_{L^{\infty} H^{1}}^{2}\left\|\Psi_{j}\right\|_{L^{\infty} H^{1}},
$$

where $1+1 / 2=1 / p+3 / r, r \in(18 / 5,6)$. Taking $\delta>0$ small enough and using the fractional Leibniz formula, we have

$$
\begin{aligned}
\left\|\left(V *|U|^{2}\right) \Psi_{j}\right\|_{\dot{H}^{\delta}} & \leq C\left(\|V\|_{p}\|U\|_{r}^{2}\left\|D^{\delta} \Psi_{j}\right\|_{r}+\|V\|_{p}\|U\|_{r}\left\|D^{\delta} U\right\|_{r}\left\|\Psi_{j}\right\|_{r}\right) \\
& \leq\|V\|_{p}\|U\|_{L^{\infty} H^{1}}^{2}\left\|\Psi_{j}\right\|_{L^{\infty} H^{1}} .
\end{aligned}
$$

This shows that the sequence $\left\{\left(V *|U|^{2}\right) \Psi_{j}\right\}_{j=0}^{\infty}$ is uniformly bounded in $H^{\delta}\left(\mathbb{R}^{3}\right)$.

Lemma 3.2. Let $T<\infty, t \in[-T, T]$, and $\varepsilon>0$. Then

$$
\left\|\int_{0}^{t} \Delta^{-1} G^{\prime}(t-s) U(s) d s\right\|_{H^{2-\varepsilon}\left(\mathbb{R}^{3}\right)} \leq C\|U\|_{L^{2}\left([-T, T], L^{2}\left(\mathbb{R}^{3}\right)\right)} .
$$


Proof (cf. [5], [10]). For $k \in \mathbb{R}$, let $F^{k}$ denote the operator on $L^{2}[-T, T]$ defined by $\widehat{F^{k} h}(n)=(i n)^{k} \widehat{h}(n), n \in \mathbb{N}-\{0\}$, and $\widehat{F^{k} h}(0)=\widehat{h}(0)$, where $h \in L^{2}[-T, T]$ and the hat denotes the Fourier transform. Making use of the discrete Plancherel identity and the transformation between time and space regularity, it follows that by taking $2 k=2-\varepsilon$ with $k<1$,

$$
\begin{aligned}
& \left\|\int_{0}^{t} \Delta^{-1} G^{\prime}(t-s) U(s) d s\right\|_{H^{2-\varepsilon}} \\
& \quad \leq\left\|\int_{-T}^{T}\left(F^{k} \chi_{[0, t]}\right)(s) \cdot\left(F^{-k} \Delta^{-1} G^{\prime}(t-\cdot) U(\cdot)\right)(s) d s\right\|_{H^{2-\varepsilon}} \\
& \leq\left\|F^{k} \chi_{[0, t]}\right\|_{L^{2}([-T, T])} \cdot\left\|F^{-k} \Delta^{-1} G^{\prime}(t-\cdot) U(\cdot)\right\|_{L^{2}\left([-T, T], H^{2-\varepsilon}\right)} \\
& \leq C\|U\|_{L^{2}\left([-T, T], L^{2}\right)},
\end{aligned}
$$

where we have used the estimate

$$
\left\|F^{k} \chi_{[0, t]}\right\|_{L^{2}([-T, T])} \leq\left(\sum_{n \in \mathbb{Z}-\{0\}}\left(n^{k} \cdot \frac{1}{n}\right)^{2}+1\right)^{1 / 2}<\infty .
$$

Now, we are in a position to prove Lemma 2.3. Note that the multilinear estimates of the two terms of $\mathcal{T}_{U_{0}}$ are similar, so we only need to estimate the first term.

STEP 1. We make use of an interior time cut-off technique to deal with the convolution kernel. It is easy to see that

$$
\begin{aligned}
& \left\|\int_{0}^{t} \Delta^{-1} G^{\prime}(t-s)\left(V *|U|^{2}\right) \Psi_{j}(s) d s\right\|_{L^{4}\left(|t|>T, H_{3}^{1}\left(\mathbb{R}^{3}\right)\right)} \\
& \leq\left\|\int_{0}^{t}|t-s|^{-1 / 2}\right\|\left(\left(V *|U|^{2}\right) \Psi_{j}\right)(s)\left\|_{H_{3 / 2}^{1}} d s\right\|_{L^{4}(|t|>T)} \\
& \leq\left\|\int_{0}^{t}|t-s|^{-1 / 2} \chi_{\{|s|>T / 2\}}(s)\right\|\left(\left(V *|U|^{2}\right) \Psi_{j}\right)(s)\left\|_{H_{3 / 2}^{1}} d s\right\|_{L^{4}(|t|>T)} \\
& \quad+\left\|\int_{0}^{t}|t-s|^{-1 / 2} \chi_{\{|s| \leq T / 2\}}(s)\right\|\left(\left(V *|U|^{2}\right) \Psi_{j}\right)(s)\left\|_{H_{3 / 2}^{1}} d s\right\|_{L^{4}(|t|>T)} \\
& =: I_{1}+I_{2} .
\end{aligned}
$$

On the one hand,

$$
\begin{aligned}
I_{1} & =\left\|\int_{0}^{t}|t-s|^{-1 / 2}\right\|\left(\left(V *|U|^{2}\right) \Psi_{j}\right)(s) \chi_{\{|s|>T / 2\}}(s)\left\|_{H_{3 / 2}^{1}} d s\right\|_{L^{4}(|t|>T)} \\
& \lesssim\left\|\left(V *|U|^{2}\right) \chi_{\{|s|>T / 2\}} \Psi_{j}\right\|_{L^{4 / 3}\left(\mathbb{R}, H_{3 / 2}^{1}\right)}
\end{aligned}
$$




$$
\begin{aligned}
\leq & \left\|\left(V *|U|^{2}\right) \chi_{\{|s|>T / 2\}}\right\|_{L^{2}\left(\mathbb{R}, L^{3}\right)}\left\|\Psi_{j}\right\|_{L^{4}\left(\mathbb{R}, H_{3}^{1}\right)} \\
& +\left\|\left(V *|U|^{2}\right) \chi_{\{|s|>T / 2\}}\right\|_{L^{2}\left(\mathbb{R}, H_{2 p}^{1}\right)}\left\|\Psi_{j}\right\|_{L^{4}\left(\mathbb{R}, L^{\widetilde{p}}\right)} \\
\leq & \left\|\Psi_{j}\right\|_{X}\left(\left\|\left(V *|U|^{2}\right) \chi_{\{|s|>T / 2\}}\right\|_{L^{2}\left(\mathbb{R}, L^{3}\right)}+\left\|\left(V *|U|^{2}\right) \chi_{\{|s|>T / 2\}}\right\|_{L^{2}\left(\mathbb{R}, H_{2 p}^{1}\right)}\right) .
\end{aligned}
$$

Since

$$
\begin{aligned}
\|\left(V *|U|^{2}\right) \chi_{\{|s|>} & T / 2\}\left\|_{L^{2}\left(\mathbb{R}, L^{3}\right)}+\right\|\left(V *|U|^{2}\right) \chi_{\{|s|>T / 2\}} \|_{L^{2}\left(\mathbb{R}, H_{2 p}^{1}\right)} \\
& \leq\|V\|_{p}\|U\|_{L^{4}\left(\mathbb{R}, L^{\widetilde{p}}\right)}^{2}+\|V\|_{p}\|U\|_{L^{4}\left(\mathbb{R}, L^{\widetilde{p}}\right)}\|U\|_{L^{4}\left(\mathbb{R}, H_{3}^{1}\right)} \\
& \leq 2\|V\|_{p}\|U\|_{L^{4}\left(\mathbb{R}, H_{3}^{1}\right)}^{2}<\infty
\end{aligned}
$$

and

$$
\begin{gathered}
\left\|\left(V *|U|^{2}\right) \chi_{\{|s|>T / 2\}}\right\|_{L^{2}\left(\mathbb{R}, L^{3}\right)}+\left\|\left(V *|U|^{2}\right) \chi_{\{|s|>T / 2\}}\right\|_{L^{2}\left(\mathbb{R}, H_{2 p}^{1}\right)} \\
=\left\|V *|U|^{2}\right\|_{L^{2}\left(|t|>T / 2, L^{3}\right)}+\left\|V *|U|^{2}\right\|_{L^{2}\left(|t|>T / 2, H_{2 p}^{1}\right)} \\
\rightarrow 0 \quad \text { as } T \rightarrow \infty
\end{gathered}
$$

where we have used the property of absolute continuity, it follows that

$$
\lim _{T \rightarrow \infty} I_{1}=0 .
$$

Similar arguments can be used to get

$$
\begin{aligned}
I_{2} & \leq\left(\int_{|t|>T}\left|\int_{\mathbb{R}}\right| t-\left.\left.s\right|^{-1 / 2} \chi_{\{|s| \leq T / 2\}}(s)\left\|\left(V *|U|^{2} \Psi_{j}\right)(s)\right\|_{H_{3 / 2}^{1}} d s\right|^{4} d t\right)^{1 / 4} \\
& \lesssim\left(\int_{T}^{\infty}\left|t-\frac{T}{2}\right|^{-\frac{1}{2} \cdot 4} d t\right)^{1 / 4}\left(\left|\int_{\mathbb{R}} \chi_{\{|s| \leq T / 2\}}(s)\left\|\left(V *|U|^{2} \Psi_{j}\right)(s)\right\|_{H_{3 / 2}^{1}} d s\right|^{4}\right)^{1 / 4} \\
& \lesssim T^{-1 / 4}\left(\int_{\mathbb{R}} \chi_{\{|s| \leq T / 2\}}(s) d s\right)^{\frac{1}{4+\varepsilon}}\left(\int_{\mathbb{R}}\left\|\left(V *|U|^{2} \Psi_{j}\right)(s)\right\|^{\frac{4+\varepsilon}{3+\varepsilon}} H_{3 / 2}^{1} d s\right)^{\frac{3+\varepsilon}{4+\varepsilon}} \\
& \lesssim T^{-\frac{\varepsilon}{4(4+\varepsilon)}}\left\|\left(\left(V *|U|^{2}\right) \Psi_{j}\right)(s)\right\|{ }_{L^{\frac{4+\varepsilon}{3+\varepsilon}}} H_{3 / 2}^{1} \\
& \leq T^{-\frac{\varepsilon}{4(4+\varepsilon)}}\left(\|V\|_{p}\left\|\Psi_{j}\right\|_{L^{4} H_{3}^{1}}\|U\|_{L^{q} L^{r}}^{2}+\|V\|_{p}\|U\|_{L^{4} H_{3}^{1}}\|U\|_{L^{q} L^{r}}\left\|\Psi_{j}\right\|_{L^{q} L^{r}}\right)
\end{aligned}
$$

where $q=4-\frac{4 \varepsilon}{8+3 \varepsilon}$ and $r=\widetilde{p}=\frac{6 p}{4 p-3}$.

For any $p \in(1,3 / 2), r \in(3,6)$, we can choose $q \in(2,4)$ such that $(q, r) \in \Lambda$, provided that $\varepsilon>0$ sufficiently small. Hence

$$
I_{2} \lesssim T^{-\frac{\varepsilon}{4(4+\varepsilon)}}\|V\|_{p}\left\|\Psi_{j}\right\|_{X}\|U\|_{X}^{2} \rightarrow 0 \quad \text { as } T \rightarrow \infty .
$$

STEP 2. In contrast to Step 1, we perform an interior cut-off in space to get 


$$
\begin{aligned}
& \left\|\int_{0}^{t} \Delta^{-1} G^{\prime}(t-s)\left(\left(V *|U|^{2}\right) \eta_{R} \Psi_{j}\right)(s) d s\right\|_{L^{4}\left(|t| \leq T, H_{3}^{1}\left(\mathbb{R}^{3}\right)\right)} \\
& \quad \lesssim\left\|\left(V *|U|^{2}\right) \eta_{R} \Psi_{j}\right\|_{L^{4 / 3}\left(|t| \leq T, H_{3 / 2}^{1}\right)} \\
& \quad \leq\left\|\left(V *|U|^{2}\right) \eta_{R}\right\|_{L^{2}\left(|t| \leq T, L^{3}\right)}\left\|\Psi_{j}\right\|_{L^{4}\left(|t| \leq T, H_{3}^{1}\right)} \\
& \quad+\left\|\left(V *|U|^{2}\right) \eta_{R}\right\|_{L^{2}\left(|t| \leq T, H_{2 p}^{1}\right)}\left\|\Psi_{j}\right\|_{L^{4}\left(|t| \leq T, L^{\widetilde{p}}\right)} \\
& \quad \leq\left\|\Psi_{j}\right\|_{X}\left(\left\|\left(V *|U|^{2}\right) \eta_{R}\right\|_{L^{2}\left(|t| \leq T, L^{3}\right)}+\left\|\left(V *|U|^{2}\right) \eta_{R}\right\|_{L^{2}\left(|t| \leq T, H_{2 p}^{1}\right)}\right)
\end{aligned}
$$

while

$$
\begin{aligned}
\left\|\left(V *|U|^{2}\right) \eta_{R}\right\|_{L^{2}\left(|t| \leq T, L^{3}\right)}+\left\|\left(V *|U|^{2}\right) \eta_{R}\right\|_{L^{2}\left(|t| \leq T, H_{2 p}^{1}\right)} \\
\quad \leq\|V\|_{p}\|U\|_{L^{4}\left(|t| \leq T, L^{\widetilde{p}}\right)}^{2}+\|V\|_{p}\|U\|_{L^{4}\left(|t| \leq T, L^{\widetilde{p}}\right)}\|U\|_{L^{4}\left(|t| \leq T, H_{3}^{1}\right)} \\
\quad \leq 2\|V\|_{p}\|U\|_{L^{4}\left(|t| \leq T, H_{3}^{1}\right)}^{2}<\infty
\end{aligned}
$$

Hence,

$$
\begin{aligned}
& \left\|\left(V *|U|^{2}\right) \eta_{R}\right\|_{L^{2}\left(|t| \leq T, L^{3}\right)}+\left\|\left(V *|U|^{2}\right) \eta_{R}\right\|_{L^{2}\left(|t| \leq T, H_{2 p}^{1}\right)} \\
& \quad=\left\|V *|U|^{2}\right\|_{L^{2}\left(|t| \leq T, L^{3}(|x|>R)\right)}+\left\|\left(V *|U|^{2}\right) \eta_{R}\right\|_{L^{2}\left(|t| \leq T, H_{2 p}^{1}(|x|>R)\right)} \\
& \quad \rightarrow 0 \quad \text { as } R \rightarrow \infty .
\end{aligned}
$$

STEP 3. Observe that for each fixed $j \in \mathbb{N}$,

$$
\lim _{M \rightarrow \infty}\left\|\mathcal{T}_{U_{0}}\left(\xi_{R} \Psi_{j}\right)\right\|_{L^{4}\left(|t| \leq T, H_{3}^{1}(|x|>M)\right)}=0 .
$$

In order to prove that the convergence is uniform in $j \in \mathbb{N}$, we use a compactness argument. By Lemma 3.1, one has

$$
\left\|\left(V *|U|^{2}\right) \xi_{R} \Psi_{j}\right\|_{H^{\delta}\left(\mathbb{R}^{3}\right)} \leq\left\|\left(V *|U|^{2}\right) \Psi_{j}\right\|_{H^{\delta}(|x| \leq 2 R)} \leq C .
$$

This together with the Rellich-Kondrashov theorem implies that $\{(V *$ $\left.\left.|U|^{2}\right) \Psi_{j}\right\}_{j=0}^{\infty}$ is compact in $L^{2}(|x| \leq 2 R)$. Hence for every $\varepsilon>0$, there is a finite set $\mathscr{A} \subset \mathbb{N}$ such that for each $j \in \mathbb{N}$, there exists $l \in \mathscr{A}$ satisfying

$$
\left\|\left(V *|U|^{2}\right) \xi_{R} \Psi_{j}-\left(V *|U|^{2}\right) \xi_{R} \Psi_{l}\right\|_{L^{2}}<\varepsilon .
$$

Hence, for $M$ large enough, by Lemma 3.2 we obtain

$$
\begin{aligned}
& \left\|\int_{0}^{t} \Delta^{-1} G^{\prime}(t-s)\left(\left(V *|U|^{2}\right) \xi_{R} \Psi_{j}\right)(s) d s\right\|_{L^{4}\left(|t| \leq T, H_{3}^{1}(|x|>M)\right)} \\
& \quad \leq\left\|\int_{0}^{t} \Delta^{-1} G^{\prime}(t-s)\left(\left(V *|U|^{2}\right) \xi_{R} \Psi_{j}-\left(V *|U|^{2}\right) \xi_{R} \Psi_{l}\right) d s\right\|_{L^{4}\left(|t| \leq T, H_{3}^{1}\right)}
\end{aligned}
$$




$$
\begin{aligned}
& +\left\|\int_{0}^{t} \Delta^{-1} G^{\prime}(t-s)\left(\left(V *|U|^{2}\right) \xi_{R} \Psi_{l}\right)(s) d s\right\|_{L^{4}\left(|t| \leq T, H_{3}^{1}(|x|>M)\right)} \\
& \leq C(T) \sup _{t \in[-T, T]} \| \int_{0}^{t} \Delta^{-1} G^{\prime}(t-s)\left(\left(V *|U|^{2}\right) \xi_{R} \Psi_{j}\right. \\
& \left.\quad-\left(V *|U|^{2}\right) \xi_{R} \Psi_{l}\right) d s \|_{H^{2-\varepsilon}\left(\mathbb{R}^{3}\right)}+\varepsilon \\
& \leq C(T) \sup _{t \in[-T, T]}\left\|\left(V *|U|^{2}\right) \xi_{R} \Psi_{j}-\left(V *|U|^{2}\right) \xi_{R} \Psi_{l}\right\|_{L^{2}\left(\mathbb{R}^{3}\right)}+\varepsilon \lesssim \varepsilon .
\end{aligned}
$$

SteP 4. After localizing $t$ and $x$ to a bounded domain, we can use the following Arzelà-Ascoli compactness argument.

Lemma 3.3. A sequence $\left\{f_{j}\right\}_{j=0}^{\infty}$ in $C\left([-T, T], H_{3}^{1}(|x| \leq M)\right)$ has a convergent subsequence iff

(i) for each $t \in[-T, T]$, the sequence $\left\{f_{j}(t)\right\}_{j=0}^{\infty}$ has a convergent subsequence in $H_{3}^{1}(|x| \leq M)$;

(ii) the sequence $\left\{f_{j}\right\}_{j=0}^{\infty}$ is equicontinuous on $[-T, T]$.

We now verify that

$$
f_{j}(t)=-\int_{0}^{t} \Delta^{-1} G^{\prime}(t-s)\left(\left(V *|U|^{2}\right) \xi_{R} \Psi_{j}\right)(s) d s
$$

satisfies the two conditions of Lemma 3.3.

By Lemma 3.2, for all $t \in[-T, T]$ we have

$$
\begin{aligned}
\| \int_{0}^{t} \Delta^{-1} G^{\prime}(t-s)\left(\left(V *|U|^{2}\right)\right. & \left.\xi_{R} \Psi_{j}\right)(s) d s \|_{H^{2-\varepsilon}(|x| \leq M)} \\
& \lesssim\left\|\left(V *|U|^{2}\right) \xi_{R} \Psi_{j}\right\|_{L^{2}\left([-T, T], L^{2}\right)} \leq C .
\end{aligned}
$$

This, together with the Rellich-Kondrashov theorem, implies that the sequence $\left\{f_{j}\right\}_{j=0}^{\infty}$ satisfies (i) of Lemma 3.3.

Next, we show the equicontinuity of the sequence $\left\{f_{j}\right\}_{j=0}^{\infty}$ on $[-T, T]$ :

$$
\begin{aligned}
\left\|f_{j}(t+h)-f_{j}(t)\right\|_{H_{3}^{1}}= & \| \int_{0}^{t+h} \Delta^{-1} G^{\prime}(t+h-s)\left(\left(V *|U|^{2}\right) \xi_{R} \Psi_{j}\right)(s) d s \\
& -\int_{0}^{t} \Delta^{-1} G^{\prime}(t-s)\left(\left(V *|U|^{2}\right) \xi_{R} \Psi_{j}\right)(s) d s \|_{H_{3}^{1}}
\end{aligned}
$$




$$
\begin{aligned}
& \leq\left\|\int_{0}^{t}\left(\Delta^{-1} G^{\prime}(t+h-s)-\Delta^{-1} G^{\prime}(t-s)\right)\left(\left(V *|U|^{2}\right) \xi_{R} \Psi_{j}\right)(s) d s\right\|_{H_{3}^{1}} \\
& \quad+\left\|\int_{t}^{t+h} \Delta^{-1} G^{\prime}(t+h-s)\left(\left(V *|U|^{2}\right) \xi_{R} \Psi_{j}\right)(s) d s\right\|_{H_{3}^{1}} \\
& =: J_{1}+J_{2} .
\end{aligned}
$$

Let $U_{j}:=\left(V *|U|^{2}\right) \xi_{R} \Psi_{j}$. By Lemma 3.2 and compactness as in Step 3,

$$
\begin{aligned}
& J_{1}=\left\|\int_{0}^{t} \Delta^{-1} G^{\prime}(t-s)(G(h)-I) U_{j}(s) d s\right\|_{H_{3}^{1}} \\
& \left.\lesssim\left\|\int_{0}^{t} \Delta^{-1} G^{\prime}(t-s)(G(h)-I) U_{j}(s) d s\right\|_{H^{2-\varepsilon}} \quad \text { for some } \varepsilon>0\right) \\
& \lesssim\left\|(G(h)-I) U_{j}\right\|_{L^{2}\left([-T, T], L^{2}\right)} \\
& \leq\left\|(G(h)-I)\left(U_{j}-U_{l}\right)\right\|_{L^{2}\left([-T, T], L^{2}\right)}+\left\|(G(h)-I) U_{l}\right\|_{L^{2}\left([-T, T], L^{2}\right)} \\
& \leq 2\left\|U_{j}-U_{l}\right\|_{L^{2}\left([-T, T], L^{2}\right)}+\left\|(G(h)-I) U_{l}\right\|_{L^{2}\left([-T, T], L^{2}\right)}<\varepsilon
\end{aligned}
$$

uniformly in $j \in \mathbb{N}$ as $|h|$ is small enough. Combining the $L^{p}-L^{p^{\prime}}$ estimate with the Hölder inequality, we deduce that

$$
\begin{aligned}
J_{2} & \leq \int_{t}^{t+h}|t+h-s|^{-1 / 2}\left\|V *|U|^{2} \Psi_{j}\right\|_{H_{3 / 2}^{1}} d s \\
& \leq\left(\int_{t}^{t+h}|t+h-s|^{-q^{\prime} / 2} d s\right)^{1 / q^{\prime}}\left\|V *|U|^{2} \Psi_{j}\right\|_{L^{q} H_{3 / 2}^{1}}
\end{aligned}
$$

where $1 / q+1 / q^{\prime}=1$.

Let $1+2 / 3=1 / p+3 / r$. One easily verifies $3<r<9 / 2$ for any $1<p<$ $3 / 2$. This allows us to choose admissible pairs $(q, r) \in \Lambda$ such that

$$
\begin{aligned}
\| V * & |U|^{2} \Psi_{j} \|_{L^{q} H_{3 / 2}^{1}} \\
& \leq\|V\|_{p}\left(\|U\|_{L^{\infty} L^{r}}^{2}\left\|\Psi_{j}\right\|_{L^{q} H_{r}^{1}}+\|U\|_{L^{\infty} L^{r}}\left\|\Psi_{j}\right\|_{L^{\infty} L^{r}}\|U\|_{L^{q} H_{r}^{1}}\right) \\
& \leq c\|V\|_{p}\|U\|_{X}^{2}\left\|\Psi_{j}\right\|_{X} \leq C
\end{aligned}
$$

and

$$
\int_{t}^{t+h}|t+h-s|^{-q^{\prime} / 2} d s \rightarrow 0 \quad \text { as } h \rightarrow 0 .
$$

Hence, $J_{2} \rightarrow 0$ uniformly on $j \in \mathbb{N}$ as $h \rightarrow 0$. 
Step 5 (A Cantor diagonal process). For each $N \in\{1,2, \ldots\}$, we choose a $T(N)$ in Step 1, an $R(N)$ in Step 2 and an $M(N)$ in Step 3 such that

$$
\begin{aligned}
& \sup _{j \in \mathbb{N}}\left\|\mathcal{T}_{U_{0}} \Psi_{j}\right\|_{L^{4}\left(|t|>T(N), H_{3}^{1}\left(\mathbb{R}^{3}\right)\right)}<1 / N, \\
& \sup _{j \in \mathbb{N}}\left\|\mathcal{T}_{U_{0}}\left(\eta_{R(N)} \Psi_{j}\right)\right\|_{L^{4}\left(|t| \leq T(N), H_{3}^{1}\left(\mathbb{R}^{3}\right)\right)}<1 / N, \\
& \sup _{j \in \mathbb{N}}\left\|\mathcal{T}_{U_{0}}\left(\xi_{R(N)} \Psi_{j}\right)\right\|_{L^{4}\left(|t| \leq T(N), H_{3}^{1}(|x|>M(N))\right)}<1 / N .
\end{aligned}
$$

In this way, we can choose inductively a subsequence $\left\{\Psi_{j, N}\right\}$ of $\left\{\Psi_{j, N-1}\right\}$, $N=1,2, \ldots$, with $\Psi_{j, 0}=\Psi_{j}$, such that $\left\{\mathcal{T}_{U_{0}}\left(\xi_{R(N)} \Psi_{j, N}\right)\right\}$ converges in $L^{4}\left(|t| \leq T(N), H_{3}^{1}(|x| \leq M(N))\right)$. Thus $\left\{\mathcal{T}_{U_{0}}\left(\xi_{R(N)} \Psi_{N, N}\right)\right\}_{N=1}^{\infty}$ converges in $L^{4}\left(\mathbb{R}, H_{3}^{1}\left(\mathbb{R}^{3}\right)\right)$. This completes the proof of Lemma 2.3 .

4. Proof of Theorem 1.1. We use the approach of Kumlin [10] and a compactness argument, which enables us to give a more concise proof.

Lemma 4.1 ([8]). Let $H$ be a Hilbert space, and let $A_{k}: H \rightarrow H, k=$ $1,2, \ldots$, be analytic mappings, uniformly bounded on all compact sets $D \subset H$. Also assume that $A_{k} u \rightarrow A u$ as $k \rightarrow \infty$ for all $u \in H$. Then the mapping $A: H \rightarrow H$ is also analytic.

According to Theorem 1.2, $\mathcal{N}(T): U_{0} \mapsto U(T)$ is analytic from $H^{1}$ to $H^{1}$ for every $T \in \mathbb{R}$. The wave operators $W_{ \pm}$and their inverses can be represented as

$$
\begin{gathered}
W_{ \pm}=\lim _{T \rightarrow \pm \infty} \mathcal{N}(-T) G(T), \\
W_{ \pm}^{-1}=\lim _{T \rightarrow \pm \infty} G(-T) \mathcal{N}(T) .
\end{gathered}
$$

Note that $\mathcal{N}(-T) G(T)$ and $G(-T) \mathcal{N}(T)$ are analytic on $H^{1}$, and $G(T)$ is isometric on $H^{1}$. Lemma 4.1 implies that $W_{ \pm}, W_{ \pm}^{-1}$ and $S$ are analytic provided that

$$
\sup _{\Phi \in D} \sup _{T \in \mathbb{R}}\|\mathcal{N}(T) \Phi\|_{H^{1}}<\infty
$$

for all compact sets $D \subset H^{1}$. In fact,

$$
\begin{aligned}
\|\mathcal{N}(T) \Phi\|_{H^{1}} \leq & \|G(T) \Phi\|_{H^{1}} \\
& +\left\|\int_{0}^{T} \Delta^{-1} G^{\prime}(t-s)\left(V *|U(\Phi)|^{2}\right) U(\Phi)(s) d s\right\|_{L^{\infty} H^{1}} \\
\leq & \|\Phi\|_{H^{1}}+\|V\|_{p}\|U(\Phi)\|_{L^{4} H_{3}^{1}}^{3} .
\end{aligned}
$$


Hence, it is enough to prove

$$
\sup _{\Phi \in D}\|U(\Phi)\|_{L^{4} H_{3}^{1}}<\infty .
$$

Indeed, as a direct result of scattering theory, we have

$$
\|U(\Phi)\|_{L^{4} H_{3}^{1}}<\infty
$$

for $\Phi \in H^{1}$. Hence, we need to prove that the bound is uniform in $\Phi \in D$.

Since $D$ is a compact subset of $H^{1}$, for fixed $0<\varepsilon_{0}<1$ there exists a finite set $\mathscr{A}=\left\{\Phi_{l_{1}}, \ldots, \Phi_{l_{n}}\right\}$ such that for any $\Phi \in D$, there exists $\Phi_{l} \in \mathscr{A}$ satisfying

$$
\left\|\Phi-\Phi_{l}\right\|_{H^{1}}<\varepsilon_{0} .
$$

Noting that $U: \Phi \mapsto U(\Phi)$ is analytic from $H^{1}$ to $L^{4} H_{3}^{1}$, we easily see that the Fréchet derivative $U^{\prime}\left(U_{l}\right)$ is a bounded operator from $H^{1}$ to $L^{4} H_{3}^{1}$. This yields

$$
\begin{aligned}
\|U(\Phi)\|_{L^{4} H_{3}^{1}} & \leq\left\|U(\Phi)-U\left(\Phi_{l}\right)\right\|_{L^{4} H_{3}^{1}}+\left\|U(\Phi)-U\left(\Phi_{l}\right)\right\|_{L^{4} H_{3}^{1}} \\
& \leq\left\|U^{\prime}\left(\Phi_{l}\right)\left(\Phi-\Phi_{l}\right)\right\|_{L^{4} H_{3}^{1}}+o\left(\varepsilon_{0}\right)+\left\|U\left(\Phi_{l}\right)\right\|_{L^{4} H_{3}^{1}} \\
& \leq C_{l}\left\|\Phi-\Phi_{l}\right\|_{H^{1}}+o\left(\varepsilon_{0}\right)+\left\|U\left(\Phi_{l}\right)\right\|_{L^{4} H_{3}^{1}} \\
& \leq C_{l} \varepsilon_{0}+o\left(\varepsilon_{0}\right)+\left\|U\left(\Phi_{l}\right)\right\|_{L^{4} H_{3}^{1}}<C .
\end{aligned}
$$

This completes the proof of Theorem 1.1.

Acknowledgments. The authors thank the referees and the associated editor for their comments and suggestions which helped improve the paper greatly. The authors are grateful to Prof. W. Strauss for his valuable suggestions. C. X. Miao was partially supported by the NSF of China (No. 10725102).

\section{References}

[1] J. Baez, Scattering and complete integrability in conformally invariant nonlinear theories, J. Math. Phys. 31 (1990), 757-762.

[2] J. Baez and Z. F. Zhou, Analyticity of scattering for the $\phi^{4}$ theory, Comm. Math. Phys. 124 (1989), 9-21.

[3] - - - Scattering and complete integrability in the massive $\phi^{4}$ theory, J. Funct. Anal. 94 (1990), 397-414.

[4] Ph. Brenner, On scattering and everywhere defined scattering operators for nonlinear Klein-Gordon equations, J. Differential Equations 56 (1985), 310-344.

[5] - On strong global solutions of nonlinear hyperbolic equations, in: Séminaire Équations aux dérivées partielles 1988-1989, exp. V, École polytechnique, Palaiseau, 1989.

[6] T. Cazenave, An Introduction to Nonlinear Schrödinger Equations, Text Met. Mat. 26, Inst. Mat., Rio de Janeiro, 1993. 
[7] J. Ginibre and G. Velo, Scattering theory in the energy space for a class of Hartree equations, in: Nonlinear Wave Equations (Providence, RI, 1998), Contemp. Math. 263, Amer. Math. Soc., Providence, RI, 2000, 29-60.

[8] E. Hille and R. S. Phillips, Functional Analysis and Semigroups, Amer. Math. Soc. Colloq. Publ. 31 (1957).

[9] M. Keel and T. Tao, Endpoint Strichartz estimates, Amer. J. Math. 120 (1998), 955-980.

[10] P. Kumlin, Analyticity of the scattering operator for the nonlinear Klein-Gordon equation with cubic nonlinearity, Comm. Math. Phys. 144 (1992), 257-278.

[11] C. X. Miao, Harmonic Analysis and Application to Differential Equations, 2nd ed., Science Press, Beijing, 2004.

Changxing Miao

Institute of Applied Physics

and Computational Mathematics

P.O. Box 8009

Beijing, China, 100088

E-mail: miao_changxing@iapcm.ac.cn

Junyong Zhang

The Graduate School of China Academy

of Engineering Physics

P.O. Box 2101

Beijing, China, 100088

E-mail: zhangjunyong111@sohu.com
Haigen Wu

The Graduate School of China Academy of Engineering Physics P.O. Box 2101 Beijing, China, 100088

and

School of Mathematics and Information Science

Henan Polytechnic University Jiaozuo, China, 454000

E-mail: wuhaigen@hpu.edu.cn

Received 1.7.2008

and in final form 17.10.2008 